\title{
Diagnostic Accuracy of Single Spot Urine for Detecting Renal Uric Acid Underexcretion in Men
}

\author{
Naoki Sakane ${ }^{\mathrm{a}, \mathrm{e}}$, Ayana Okuno ${ }^{\mathrm{b}}$, Kouji Nomaguchi ${ }^{\mathrm{b}}$, Miyuki Tanaka ${ }^{\mathrm{b}}$, Fumiaki Abe ${ }^{\mathrm{b}}$, \\ ${\text { Izumi Kakiuchic }{ }^{c} \text {, Kyoko Kiyosawa }}^{c}$, Mitsunaga Miyasakac ${ }^{c}$, Masahiko Nakamura ${ }^{d}$
}

\begin{abstract}
Background: The uric acid (UA) clearance test to evaluate the hyperuricemia phenotype requires a great deal of time. However, the utility of single spot urine is scarce. The study aimed to determine the diagnostic accuracy of single spot urine for predicting renal UA underexcretion (the decreased UA excretion) in men.

Methods: A total of 73 male participants aged 20 - 74 years with a UA level of $6.0-7.9 \mathrm{mg} / \mathrm{dL}$ were enrolled in the study. Renal UA underexcretion was defined as $<7.3 \mathrm{~mL} / \mathrm{min}$ using the 60 -min method. Urinary UA to creatinine ratio (UACR), fractional clearance of urate (FCU), and the Simkin index were calculated. A receiver operating characteristic (ROC) analysis was performed to compare the diagnostic utility of these parameters for predicting UA underexcretion.
\end{abstract}

Results: In the ROC analysis, the area under the curve values of the UACR, FCU, and the Simkin index for predicting UA underexcretion were 0.903 (95\% confidence interval (CI): 0.830 - 0.976), 0.841 (95\% CI: 0.749 - 0.933), and 0.779 (95\% CI: 0.673 - 0.885), respectively. An optimal UACR cutoff of 0.460 (sensitivity $89.2 \%$, specificity $80.6 \%$, overall diagnostic accuracy $84.9 \%$, positive predictive value $82.5 \%$, and negative predictive value $87.9 \%$ ) was identified.

Conclusions: These results suggest that the UACR is a simple and efficient test with high sensitivity and specificity levels for predicting renal UA underexcretion in men.

Keywords: Uric acid to creatinine ratio; Fractional clearance of

Manuscript submitted June 8, 2020, accepted June 16, 2020

Published online June 25, 2020

aDivision of Preventive Medicine, Clinical Research Institute, National Hospital Organization Kyoto Medical Center, Kyoto, Japan

${ }^{b}$ Food Ingredients \&Technology Institute, R\&D Division, Moringa Milk Insustry Co., Ltd. Kanagawa, Japan

'Department of Nursing, Matsumoto Junior College, Matsumoto, Nagano, Japan

dMatsumoto City Hospital, Matsumoto, Nagano, Japan

${ }^{\text {e}}$ Corresponding Author: Naoki Sakane, Division of Preventive Medicine, Clinical Research Institute, National Hospital Organization Kyoto Medical Center, 1-1 Mukaihata-cho, Fukakusa, Fushimi-ku, Kyoto 612-8555, Japan. Email: nsakane@gf6.so-net.ne.jp

doi: https://doi.org/10.14740/jocmr4250 urate; Simkin index; Diagnostic accuracy

\section{Introduction}

Hyperuricemia is a major risk factor for gout and is also independently associated with cardiovascular disease [1]. Moreover, hyperuricemia is a common disease that can be treated by general and family physicians, with a wide range of diagnoses and treatment options [2]. The hyperuricemia pathogenesis consists of overproduction and underexcretion, which can be estimated using the uric acid (UA) clearance (Cua) test. As a result, hyperuricemia was classified into four types: overproduction, underexcretion, combined, and normal type [3]. However, the Cua test is a time-consuming method as urine volume should be measured on time in primary care settings. Therefore, the Simkin index $[4,5]$ and fractional clearance of urate (FCU) $[6,7]$ have been used in physiological studies but usually employ 60 -min or $24-\mathrm{h}$ urine collections. The urinary UA to creatinine ratio (UACR) was used as a screening test for congenital purine metabolism disorders $[8,9]$. However, research evidence between the UACR and renal UA underexcretion has been scarce. This study aimed to determine the diagnostic accuracy of single spot urine for predicting renal UA underexcretion in men.

\section{Materials and Methods}

\section{Participants}

This study was performed using baseline data from a randomized controlled trial according to the Standards for the Reporting of Diagnostic Accuracy Studies (STARD) reporting guidelines [10]. The participants were recruited through flyers and word of mouth in Matsumoto City, Nagano Prefecture, Japan. The inclusion criteria were: 1) Male subjects aged 20 74 years old; and 2) Subjects who showed a serum UA (SUA) level of $6.0-7.9 \mathrm{mg} / \mathrm{dL}$. The exclusion criteria included: 1) Subjects who regularly consume food or supplement improving the UA level; 2) Subjects who are currently receiving treatment for hyperuricemia or gout; 3) Subjects who are under treatment 
or have a medical history of disease for gout, urinary stone, kidney stone, and rheumatoid arthritis; 4) Subjects who have serious disease or medical history in the brain, liver, kidney, heart, lung, gastrointestinal tract, or blood; 5) Subjects who excessively drink alcohol (alcohol conversion over $60 \mathrm{~g}$ /day); 6) Subjects who had a medical history of serious allergy to medicine or food; 7) Participants in other clinical trials; and 8) Subjects who were judged unsuitable for participating in this study based on subject background, physical finding, and interview by physician. The study was approved by the Matsumoto Junior College Ethics Committee, Japan. This trial was registered with the University Hospital Medical Information Network (UMIN000039465). This study was conducted in compliance with the ethical standards of the responsible institution on human subjects as well as with the Helsinki Declaration.

\section{Cua test}

The Cua test was performed according to the Japanese guidelines for the hyperuricemia and gout management $[11,12]$. Cua was measured using the 60 -min method. Briefly, patients ate low purine diets for 3 days before the start of the test, fasted on the day of the test, and voided at 30 min after drinking 300 $\mathrm{mL}$ of water. Urine was collected during a $60-\mathrm{min}$ period, and a blood sample was obtained at the midpoint of urine collection. Renal UA underexcretion was defined as $<7.3 \mathrm{~mL} / \mathrm{min}$ of Cua. Urine uric acid (UUA) per urine creatinine (UCr) rates were calculated using the formula: UUA/UCr. FCU was calculated using the formula: $\mathrm{FCU}=(\mathrm{UUA} \times$ serum creatinine $(\mathrm{SCr})) /$ $(\mathrm{SUA} \times \mathrm{UCr})$. In contrast, the Simkin index does not include plasma UA concentrations: Simkin index $=\mathrm{UUA} \times \mathrm{SCr} / \mathrm{UCr}$.

\section{Measures}

Height, body weight, body mass index (BMI), alcohol consumption, aspartate aminotransferase (AST), alanine aminotransferase (ALT), $\gamma$-glutamyl transferase (GGT), $\mathrm{SCr}$, SUA, UCr, and UUA were measured. The formula for BMI is weight in kilograms divided by height in meters squared. Estimated glomerular filtration rate (eGFR) was calculated using an equation designed for the Japanese subjects, as previously described [13].

\section{Sample size}

Sample size was estimated using the easy ROC: a web tool for ROC curve analysis (version 1.3) (http://www.biosoft.hacettepe.edu.tr/easyROC/). We estimated that a sample size of 66 participants would allow us to estimate areas under the curve (AUCs) of $0.7,80 \%$ power, and allocation ratio of $1: 1$.

\section{Statistical analysis}

Receiver operating characteristic (ROC) curve analysis was used to determine the optimal cutoff points of the parameters for detecting renal UA underexcretion. The AUC with confidence intervals (CIs) was also presented. The AUC values of $\geq 0.90$ are considered excellent, values between 0.80 - 0.89 are deemed good, values $0.70-0.79$ are fair, and those $<0.70$ are considered poor $[14,15]$. The Youden index was used to identify the optimal cutoff value for detecting renal UA underexcretion [16]. The AUC values were adjusted for age, BMI, and logarithm of alcohol consumption. Sensitivity, specificity, positive predictive value, negative predictive value, and diagnostic accuracy were calculated. The distribution of the different variables was examined for normality using the Kolmogorov-Smirnov test. Data were expressed as mean \pm standard deviation or percentage. All normally distributed data were analyzed using the Student's $t$-test to evaluate differences in mean and Chi-square test and evaluate differences in proportions. Data found to be non-normally distributed were analyzed using the Mann-Whitney U test for independent subgroups and the Wilcoxon test for dependent subgroups. In order to identify significant differences between groups after $\chi^{2}$ tests, we consecutively carried out a residual error analysis. A $P$ value of less than 0.05 was deemed statistically significant.

\section{Results}

\section{Clinical characteristics of patients with renal UA under- excretion}

The UA underexcretion prevalence was 54.8\%. The participants with renal UA underexcretion had a higher age than those without, although they had lower eGFR, UACR, FCU, and Simkin index (Table 1). There was no difference in alcohol consumption and medication between groups.

\section{Diagnostic performance of the parameters in predicting renal UA underexcretion}

In the ROC analysis, the AUC values of the UACR, FCU, and Simkin index for predicting renal UA underexcretion were 0.903 (95\% CI: 0.830 - 0.976), 0.841 (95\% CI: 0.749 - 0.933), and 0.779 (95\% CI: 0.673 - 0.885), respectively (Fig. 1). An optimal UACR cutoff was 0.460 (sensitivity $89.2 \%$, specificity $80.6 \%$, overall diagnostic accuracy $84.9 \%$, positive predictive value $82.5 \%$, and negative predictive value $87.9 \%$ ). The UACR and FCU were superior to the Simkin index for predicting renal UA underexcretion $(\mathrm{P}=0.004$ and $\mathrm{P}=0.013$, respectively) (Table 2). After adjusting for age, BMI, and logarithm of alcohol consumption, the AUC values of the UACR, FCU, and Simkin index for predicting renal UA underexcretion were 0.936 (95\% CI: 0.875 - 0.997), 0.911 (95\% CI: 0.842 - 0.981), and 0.874 (95\% CI: 0.790 - 0.959), respectively.

\section{Discussion}

This study demonstrated that the UACR was an excellent pre- 
Table 1. Clinical Characteristic of the Participants With or Without Renal UA Underexcretion

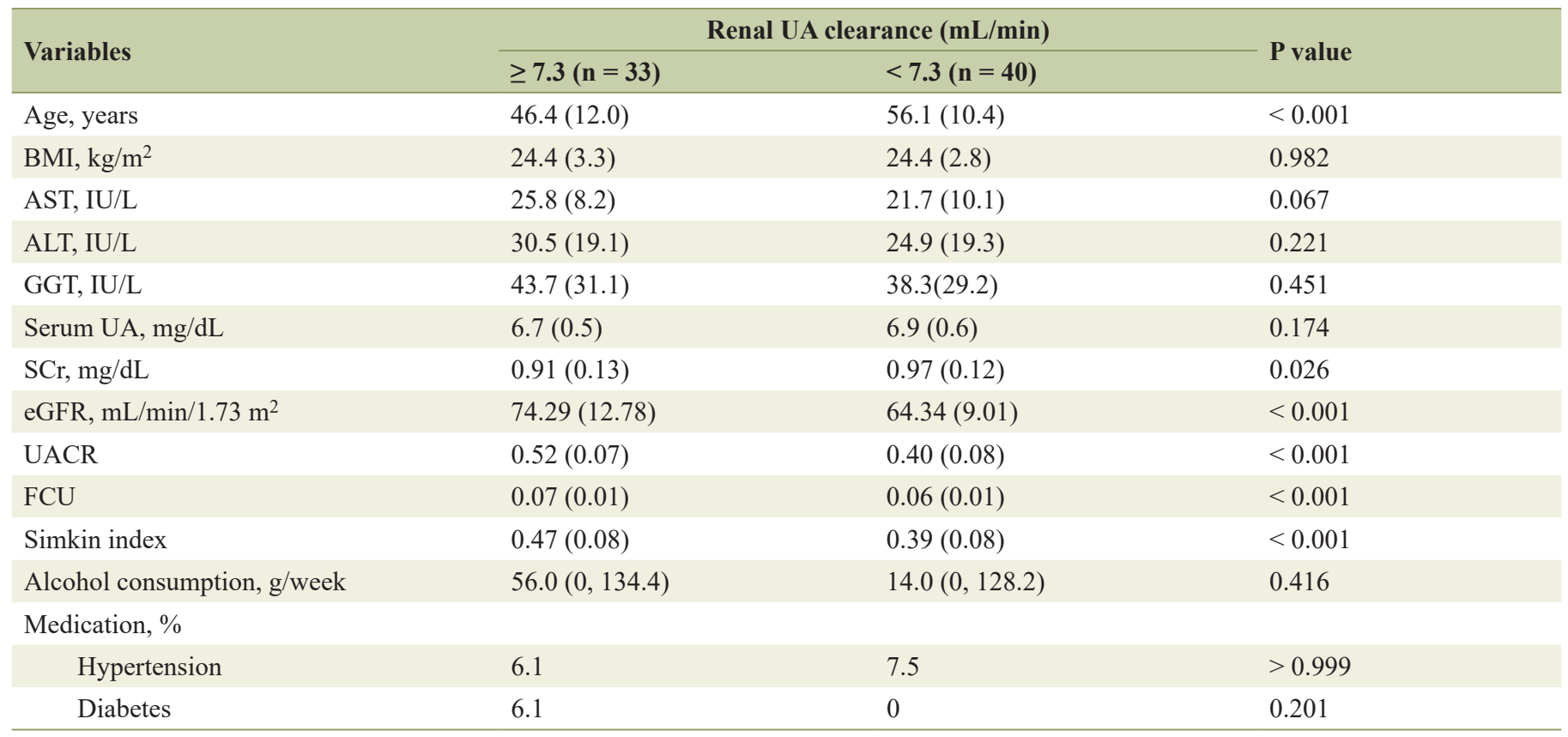

Mean (standard deviation), median (25\%, $75 \%)$, or \%. BMI: body mass index; AST: aspartate aminotransferase; ALT: alanine transferase; GGT: Y-glutamyl transferase; UA: uric acid; SCr: serum creatinine; eGFR: estimated glomerular filtration rate; UACR: urinary uric acid to creatinine ratio; FCU: fraction clearance of urate.

dictor of renal UA underexcretion. Thus, the UACR was found to be a simple, rapid, low-cost, and reliable test for UA underexcretion screening. The UA underexcretion prevalence was $58.9 \%$ in this study. The value was lower than the previous study $(85 \%)$ [17]. These differences might account for the hyperuricemia or gout severity.

FCU showed good accuracy, although the Simkin index was fairly accurate in detecting renal UA underexcretion.

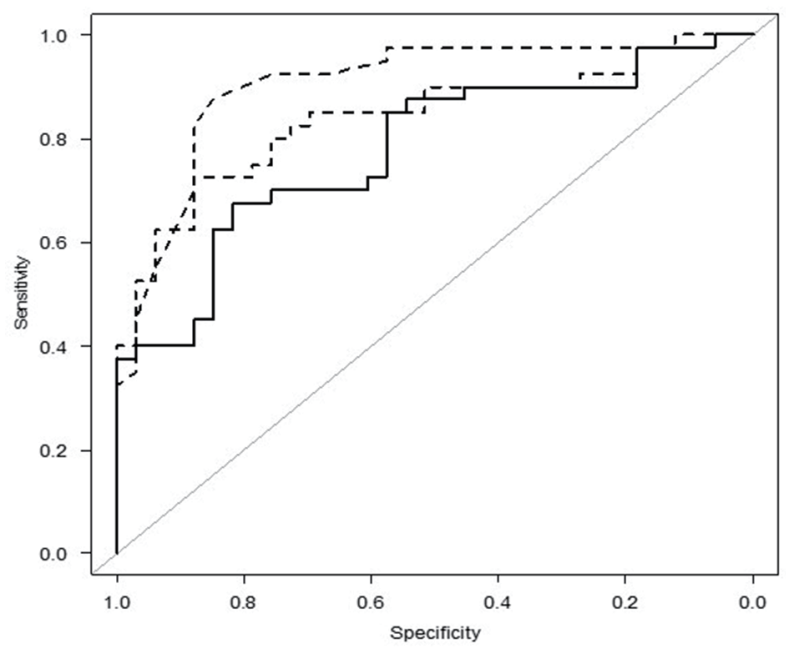

Figure 1. The ROC curve of the UACR, FCU, and the Simkin index for predicting renal UA underexcretion. ROC: receiver operating characteristic; UACR: urinary uric acid to creatinine ratio; FCU: fractional clearance of urate.
Measurement of urine volume is not required, which is a significant practical advantage. Graessler et al used 0.06 as the lower limit of normal [18]. In this study, we also identified an optimal cutoff point of 0.06 for FCU, which is consistent with a previous study [18]. Further examination is required to confirm these issues in different populations.

Simkin et al [4] proposed a spot morning urine test of urate excretion normalized to GFR to identify UA overproducers. In this study, we also collected urine samples in the morning. Thus, the timing of urine sample collection is desirable in the morning after overnight fasting and drinking of water.

\section{Strengths and limitations}

The strengths of the present study include STARD guidelinebased research, and sufficient sample size. The limitations of the present study include only male participants and no patients with UA levels of $\geq 8.0 \mathrm{mg} / \mathrm{dL}$ or gout. Generalizability was limited because of our findings in men with UA of 6.0 - $7.9 \mathrm{mg} / \mathrm{dL}$. Subjects with SUA levels of $\geq 6.0 \mathrm{mg} / \mathrm{dL}$ had a significantly increased risk for kidney impairment [19]. Guideline documents were concordant and recommended a target for SUA $<6.0 \mathrm{mg} / \mathrm{dL}$ for long-term control [20]. A simple management recommendation of a 6-7-8 rule was proposed through the consensus of expert physicians [21]. People with these UA ranges were the target for the non-pharmacological approach [22]. We recruited the participants for the study of foods with health claims preventing hyperuricemia and gout. Further researches including patients with UA levels of $\geq 8.0$ $\mathrm{mg} / \mathrm{dL}$ or gout are required. Smoking, alcohol drinking, and 
Table 2. Comparison of the UACR, FCU and the Simkin Index for Predicting Renal UA Underexcretion

\begin{tabular}{lllllllllllll} 
Variables & Cutoff & Sn, \% & Sp, \% & PPV, \% & NPV, \% & DA, \% & +LR & -LR & AUC (95\% CI) & P value $^{\mathbf{a}}$ & P value $^{\mathbf{b}}$ \\
\hline UACR & 0.460 & 89.2 & 80.6 & 82.5 & 87.9 & 84.9 & 4.59 & 0.13 & $0.903(0.830-0.976)$ & 0.158 & 0.004 & 0.749 \\
FCU & 0.060 & 67.5 & 87.9 & 87.1 & 69.0 & 76.7 & 5.57 & 0.37 & $0.841(0.749-0.933)$ & - \\
Simkin index & 0.399 & 81.2 & 65.9 & 65.0 & 81.8 & 72.6 & 2.38 & 0.29 & $0.779(0.673-0.885)$ & 0.013 & - \\
\hline
\end{tabular}

$\mathrm{UACR}=\mathrm{UUA} / \mathrm{UCr} ; \mathrm{FCU}=(\mathrm{UUA} \times \mathrm{SCr}) /(\mathrm{SUA} \times \mathrm{UCr})$; Simkin index $=\mathrm{UUA} \times \mathrm{SCr} / \mathrm{UCr}$. ${ }^{\mathrm{a} v s}$. FCU; ${ }^{b} \mathrm{vs}$. Simkin index. UACR: urinary uric acid to creatinine ratio; FCU: fraction clearance of urate; Sn: sensitivity; Sp: specificity; PPV: positive predictive value; NPV: negative predictive value; DA: diagnostic accuracy; +LR: positive likelihood ratio; -LR: negative likelihood ratio; AUC: area under the curve; UUA: urine uric acid; UCr: urine creatinine; SCr: serum creatinine.

other pharmacotherapy affecting insulin sensitivity are informative because these factors can influence the reuptake of UA in renal tubule. After adjusting for age, BMI, and alcohol consumption, the similar results were obtained. But we do not have the data of smoking habits. Careful attention should be paid to interpret the results.

We adopted the 60-min method instead of the 24-h method in this study. We do not know the diagnostic accuracy of UACR for the results using the 24-h method. The measurement of 24-h urinary UA excretion is frequently used to evaluate disease status and select drugs that lower the SUA levels. However, the 24-h urine collection is cumbersome and inconvenient, and sometimes unreliable because of incomplete sampling. Choi et al reported that there was a good correlation between the random urinary UACR and 24-h UUA excretion [23].

Reduced urinary UA excretion was observed in patients with proteinuria or metabolic syndrome [24, 25]. Further examination including proteinuria or metabolic syndrome is required to confirm these issues in the future.

In conclusion, the present study indicates that the UACR is a convenient, valid, and reliable indicator for predicting renal UA underexcretion. The UACR may be applied in primary care settings when they do not perform the 60 -min method.

\section{Acknowledgments}

We thank all the study investigators and staff and participants who participated in this study for helpful discussions during manuscript development.

\section{Financial Disclosure}

Financial support for the clinical research was provided Morinaga Milk Industry, Ltd. (Tokyo, Japan). This manuscript was reviewed by the funding company. This work was partly supported by JSPS KAKENHI (Grant Number 18K01988).

\section{Conflict of Interest}

None to declare.

\section{Informed Consent}

Informed written consent was obtained from participants.

\section{Author Contributions}

NS, AO, KN, MT, IK, KK, MM, MN, and FA conceived and designed the study. NS analyzed the data. AO, KN, MT, and FA contributed to participants' data collection. IK, KK, and MM contributed to ethical committee approval. NS wrote the paper. All authors read and approved the final version of the manuscript.

\section{Data Availability}

The authors declare that data supporting the findings of this study are available within the article.

\section{References}

1. Lim SS, Yang YL, Chen SC, Wu CH, Huang SS, Chan WL, Lin SJ, et al. Association of variability in uric acid and future clinical outcomes of patient with coronary artery disease undergoing percutaneous coronary intervention. Atherosclerosis. 2020;297:40-46.

2. Yamanaka H, Metabolism TG. Essence of the revised guideline for the management of hyperuricemia and gout. Japan Med Assoc J. 2012;55(4):324-329.

3. Okui D, Sasaki T, Fushimi M, Ohashi T. The effect for hyperuricemia inpatient of uric acid overproduction type or in combination with topiroxostat on the pharmacokinetics, pharmacodynamics and safety of dotinurad, a selective urate reabsorption inhibitor. Clin Exp Nephrol. 2020;24(Suppl 1):92-102.

4. Simkin PA, Hoover PL, Paxson CS, Wilson WF. Uric acid excretion: quantitative assessment from spot, midmorning serum and urine samples. Ann Intern Med. 1979;91(1):44-47.

5. Indraratna PL, Stocker SL, Williams KM, Graham GG, Jones G, Day RO. A proposal for identifying the low renal uric acid clearance phenotype. Arthritis Res Ther. 2010;12(6):149.

6. Perez-Ruiz F, Calabozo M, Erauskin GG, Ruibal A, 
Herrero-Beites AM. Renal underexcretion of uric acid is present in patients with apparent high urinary uric acid output. Arthritis Rheum. 2002;47(6):610-613.

7. Vitart V, Rudan I, Hayward C, Gray NK, Floyd J, Palmer $\mathrm{CN}$, Knott SA, et al. SLC2A9 is a newly identified urate transporter influencing serum urate concentration, urate excretion and gout. Nat Genet. 2008;40(4):437-442.

8. Kaufman JM, Greene ML, Seegmiller JE. Urine uric acid to creatinine rtio - a screening test for inherited disorders of purine metabolism. Phosphoribosyltransferase (PRT) deficiency in X-linked cerebral palsy and in a variant of gout. J Pediatr. 1968;73(4):583-592.

9. Wortmann RL, Fox IH. Limited value of uric acid to creatinine ratios in estimating uric acid excretion. Ann Intern Med. 1980;93(6):822-825.

10. Cohen JF, Korevaar DA, Altman DG, Bruns DE, Gatsonis CA, Hooft L, Irwig L, et al. STARD 2015 guidelines for reporting diagnostic accuracy studies: explanation and elaboration. BMJ Open. 2016;6(11):e012799.

11. Yamanaka H, Japanese Society of G, Nucleic Acid M. Japanese guideline for the management of hyperuricemia and gout: second edition. Nucleosides Nucleotides $\mathrm{Nu}-$ cleic Acids. 2011;30(12):1018-1029.

12. Saito J, Matsuzawa Y, Ito H, Omura M, Kino T, Nishikawa T. Alkalizer administration improves renal function in hyperuricemia associated with obesity. Jpn Clin Med. 2013;4:1-6.

13. Matsuo S, Imai E, Horio M, Yasuda Y, Tomita K, Nitta $\mathrm{K}$, Yamagata $\mathrm{K}$, et al. Revised equations for estimated GFR from serum creatinine in Japan. Am J Kidney Dis. 2009;53(6):982-992.

14. Hanley JA, McNeil BJ. The meaning and use of the area under a receiver operating characteristic (ROC) curve. Radiology. 1982;143(1):29-36.

15. Metz CE. Basic principles of ROC analysis. Semin Nucl Med. 1978;8(4):283-298.

16. Fluss R, Faraggi D, Reiser B. Estimation of the Youden Index and its associated cutoff point. Biom J.
2005;47(4):458-472.

17. Nakamura $T$, Takagi $K$, Ueda T. [Dynamics of uric acid metabolism in hyperuricemia]. Nihon Rinsho. 1996;54(12):3230-3236.

18. Graessler J, Graessler A, Unger S, Kopprasch S, Tausche AK, Kuhlisch E, Schroeder HE. Association of the human urate transporter 1 with reduced renal uric acid excretion and hyperuricemia in a German Caucasian population. Arthritis Rheum. 2006;54(1):292-300.

19. Toyama T, Furuichi K, Shimizu M, Hara A, Iwata Y, Sakai N, Perkovic V, et al. Relationship between serum uric acid levels and chronic kidney disease in a Japanese Cohort with normal or mildly reduced kidney function. PLoS One. 2015;10(9):e0137449.

20. Li Q, Li X, Wang J, Liu H, Kwong JS, Chen H, Li L, et al. Diagnosis and treatment for hyperuricemia and gout: a systematic review of clinical practice guidelines and consensus statements. BMJ Open. 2019;9(8):e026677.

21. Nakajima H. [Management of hyperuricemia in occupational health: with reference to "guidelines for the management of hyperuricemia and gout"]. Sangyo Eiseigaku Zasshi. 2003;45(1):12-19.

22. Kakutani-Hatayama M, Kadoya M, Okazaki H, Kurajoh M, Shoji T, Koyama H, Tsutsumi Z, et al. Nonpharmacological management of gout and hyperuricemia: hints for better lifestyle. Am J Lifestyle Med. 2017;11(4):321-329.

23. Choi ST, Moon SJ, Kang EJ. Random urinary uric acid/ creatinine ratio is useful in the estimation of 24-hour urine uric acid excretion in patients with gout [abstract]. Arthritis Rheumatol. 2017;69(suppl 10).

24. Zou H, Xiang M, Ye X, Xiong Y, Xie B, Shao J. Reduction of urinary uric acid excretion in patients with proteinuria. J Chromatogr B Analyt Technol Biomed Life Sci. 2015;1006:59-64.

25. Fraile JM, Puig JG, Torres RJ, de Miguel E, Martinez P, Vazquez JJ. Uric acid metabolism in patients with primary gout and the metabolic syndrome. Nucleosides Nucleotides Nucleic Acids. 2010;29(4-6):330-334. 